\title{
The Effect of the MTHFR C677T Mutation on Athletic Performance and the Homocysteine Level of Soccer Players and Sedentary Individuals
}

\author{
by \\ Nurten Dinç¹, Selda Bereket Yücel², Fatma Taneli, Metin Vehbi Sayın ${ }^{1}$
}

This study investigated athletic performance and homocysteine (Hcy) levels in relation to the methylenetetrahydrofolate reductase (MTHFR) C677T mutation and explored the relationship between this mutation and other cardiac risk factors in soccer players and sedentary individuals. The study groups consisted of randomly selected soccer players $(n=48)$ from the Turkish Super and Major League and sedentary male students $(n=48)$ aged 18 27. Anthropometric variables, aerobic and anaerobic thresholds were measured, furthermore, biochemical assays were performed. The level of HDL cholesterol, LDL cholesterol, triglyceride, Hcy, folate, vitamin B12, hemogram and MTHFR C677T was investigated. The results showed that there was a statistical difference between the two groups in terms of body mass, body fat, the BMI, the aerobic threshold heart rate (ATHR), aerobic threshold velocity (ATVL) and anaerobic threshold velocity (ANTVL). The soccer players were found to have lower levels of triglyceride, total cholesterol and LDL cholesterol, and higher levels of folate than the sedentary participants. The analysis of the alleles of the MTHFR C677T polymorphism showed that the participants that carried TT genotypes had a lower level of vitamin $B 12$ and folate, and a higher level of Hcy than the participants carrying CC and CT genotypes. In conclusion, the baseline homocysteine and cardiovascular fitness levels of healthy young males with the TT genotypes of the MTHFR C677T genotype were found to strongly correlate with their levels of Hcy.

Key words: MTHFR C677T, soccer, homocysteine, athletic performance.

\section{Introduction}

Homocysteine (Hcy) is a sulfur-containing amino acid found in nutritional protein and formed as an intermediate product of the methionine metabolism. Elevated plasma total Hcy concentration is an independent risk factor for coronary ischemic diseases, a stroke, vascular diseases and venous thrombosis (Husemoen et al., 2006; Mennen et al., 2002; Rasmussen et al., 2000). Life factors that were found to affect Hcy levels include age, sex, diet, plasma folate, vitamin B6, vitamin B12, smoking, consumption of coffee, tea and alcohol, and physical activity (Rasmussen et al., 2000; Husemoen et al., 2006).
In addition to these important factors, there is the 5,10-methylenetetrahydrofolate reductase enzyme (MTHFR, EC 1.5.1.20) which is the genetic marker of the Hcy metabolism (Husemoen et al., 2004). The MTHFR enzyme is associated with an elevated total plasma Hcy concentration and an increased cardiovascular risk. MTHFR plays an important role in the formation of the methyl donor 5-methyltetrahydrofolate for the DNA methylation and remethylation of Hcy to methionine and Sadenosyl methionine (Andreassi et al., 2003). There are 14 mutations of MTHFR. One important

1 - Celal Bayar University, School of Physical Education and Sports, Department of Kinesiology and Training.

2- Marmara University, School of Physical Education and Sports, Department of Kinesiology and Training.

3. Celal Bayar University, Faculty of Medicine, Department of Biochemistry. 
mutation is the C677T polymorphism (Weisberg et al., 1998) which is associated with reduced MTHFR enzyme activity (Andreassi et al., 2003). The 5,10-methylene-tetrahydrofolate enzyme bound to flavin adenine dinucleotide (FAD) irreversibly catalyzes the formation of 5methyltetrahydrofolate from the 5,10methyltetrahydrofolate, the methyl donor in the Hcy methionine remethylation (Klerk et al., 2002; Ueland et al., 2001). This enzyme is positioned at a metabolic crossroads and directs the folate pool in the metabolism towards Hcy remethylation rather than the DNA and RNA biosynthesis. The enzyme activity is reduced in individuals with C$\mathrm{T}$ substitution in the 677 gene and these individuals have a higher level of Hcy (Fletcher and Kessling, 1998) and a lower level of folate (Klerk et al., 2002).

People with the homozygote TT genotype have a higher level of plasma Hcy than those with the heterozygote $\mathrm{CT}$ or homozygote $\mathrm{CC}$ genotype (Pullin et al., 2002). However, the relationship between the MTHFR polymorphism and vitamin B and total Hcy can vary. Vitamin deficiency is associated with a higher level of Hcy in people carrying the TT genotype than those carrying the CC or CT genotype (Husemoen, 2004). Andreassi et al. (2003) compared the CC and CT genotypes with the TT genotype in 44 angiographic patients and found higher Hcy levels in the TT genotype. In a study by Klerk et al. (2002), the experimental group consisted of 11,162 participants and the control group of 12,758 . For both groups, participants carrying the CC genotype were found to have a higher level of Hcy and a lower level of folate than those carrying the TT or CT genotype.

Fortunato et al. (2007) conducted a study with 54 male and 46 female participants aged from 20 to 30, who exercised 3 to 4 times a week. Significant differences were found between males and females in relation to the levels of creatine, hemoglobin, hematocrit, HDL cholesterol, triglyceride and fibrinogen. Of the females who exercised regularly, those carrying the homozygote TT genotype of the MTHFR C677T polymorphism were found to have a lower level of hemoglobin than those carrying the CC or CT genotype. Dankner et al. (2007) found that Hcy levels were significantly higher in the sedentary population than in subjects who participated in regular physical activity. In both genders, the Hcy levels of the participants were lower in those who carried the CC homozygote genotype when compared to those carrying the CT and TT genotype. In terms of physically active subjects, a negative correlation was only found between Hcy levels in the homozygote TT genotype of the MTHFR C677T polymorphism.

These results indicated that age, sex, diet and different types and intensity of exercise have distinct effects on the independent cardiovascular risk factors, particularly on the Hcy metabolism. The effects of these variables can vary with the unique genetic make-up of an individual. Therefore, the primary objective of this study was to investigate athletic performance and homocysteine levels in relation to the MTHFR C677T mutation and to explore the relationship between this mutation and other cardiac risk factors. The secondary objective was to determine which alleles of the MTHFR C677T polymorphism were associated with high levels of Hcy, total cholesterol, LDL, HDL, triglyceride and hemoglobin.

\section{Material and Methods}

\section{Participants}

The participants in the study consisted of 96 randomly selected men $(n=96)$ aged from 18 to 27 . Half the participants $(n=48)$ played soccer in an A2 team of the Turkcell Super League and Bank Asya Major League in Turkey. The remaining 48 participants were students from the School of Physical Education and Sports. The study was approved by the Ethics Committee at the Faculty of Medicine, Celal Bayar University. All the participants completed a medical history inventory form and signed the informed consent form that explained the objectives and risks of the study. None of the participants had a history of hypertension, coronary heart disease, anemia and diabetes.

\section{Measures}

Anthropometric measurements, aerobic threshold (AT), anaerobic threshold (ANT) and biochemical blood tests were obtained from all the participants and analyzed. The biochemical tests were conducted from venous blood samples taken from the participants between 08:00 and 10:00 a.m. following a $12 \mathrm{~h}$ fast. The biochemical tests consisted of the analysis of total cholesterol, HDL cholesterol, LDL cholesterol, triglyceride, Hcy, 
folate, vitamin B12, hemogram and MTHFR C677T mutation.

Body mass and fat free mass (FFM) were measured using Tanita Bioelectrical Impedance Analysis technology (Tanita 300 MA, Tanita C.O., Tokyo - Japan). The body mass index was calculated from the participants' height and body mass according to the following formula: $\mathrm{BMI}=$ Body mass (kg) / [height (m)] 2.

\section{Measurement of Aerobic Threshold and Anaerobic Threshold Rates}

The test protocol (Borch, 1993) comprised a standard warm-up at $8 \mathrm{~km} / \mathrm{h}$ on a $20 \times 40$ rectangular grass field followed by an exercise consisting of 5 min laps, each lap followed by an increase in pace of $1.2 \mathrm{~km} / \mathrm{h}$ with 1 min passive rest intervals, until voluntary exhaustion, after which there was $15 \mathrm{~min}$ of passive rest. Then, the participants completed a $40-\mathrm{m}$ shuttle run with their speed monitored using an audio signal every $20 \mathrm{~m}$. If a participant missed the first signal and caught the second, the test continued. If a participant missed both signals and was too tired to maintain the pace, the test was terminated for that participant. Following each lap, the participant's heart rate (HR) was monitored using an HR monitor (Sportstester PE300, Germany), and blood samples were taken from their fingertip to measure lactate concentration. The total duration of the test including shuttle runs and rest periods in which blood lactate measurements were taken, lasted 30 to $40 \mathrm{~min}$.

Total blood lactate was measured using a YSI 1500 SPORT model lactate analyzer (Yellow Springs Instruments Incorp, USA). The AT and ANT rates $(\mathrm{km} / \mathrm{h})$ were calculated from the ratelactate diagram using interpolation or extrapolation. The calculation of the AT and ANT rates, values of the $\mathrm{HR}$ and lactate recorded following each lap were entered in the diagram corresponding to the rate in a given lap. The rate corresponding to the $2 \mathrm{mM}$ lactate value was ATVL, the heart rate corresponding to this value was the ATHR, the rate corresponding to the 4 $\mathrm{mM}$ lactate value was ANTVL and the heart rate corresponding to this value was the anaerobic threshold heart rate (ANTHR).

\section{Blood Collection and Biochemical Analysis}

Following a $12 \mathrm{~h}$ fast, between 08:00 and 10:00 a.m blood was collected from the veins of the forearm of the participants, and stored in a red top test tube (flat tube) and a purple test tube (EDTA-containing tube). The red test tube that did not contain anticoagulant was used for the analysis of serum total cholesterol, triglyceride, HDL cholesterol, Hcy, folate and vitamin B12. The blood was centrifuged and the serums were separated from the cells to be analyzed. The blood specimen in the purple test tube containing the hemogram EDTA was analyzed on the same day using the blood cell count machine. For the MTHFR C677T mutation and analysis, DNA samples were obtained from the EDTA-containing blood samples through DNA extraction using DNA isolation kits, and stored at $-80^{\circ} \mathrm{C}$. The DNA samples were amplified using a thermal cycler (Applied Biosystems 2720, USA).

\section{Statistical Analysis}

The statistical analyses were performed with the SPSS 15 package program, working under Windows XP. A Student's $t$ test was used to evaluate the probable difference between the independent variables. A Kruskall Wallis analysis was used to determine the statistical difference between the physical, physiological and anthropometric measurements of the participants according to the alleles of the MTHFR C677T polymorphism. The significance level used throughout the study was set at 0.05 .

\section{Results}

The comparison of the results of the two groups from the Student's $t$ test showed a statistical difference in terms of age $(\mathrm{p}=0.01)$, body mass $(\mathrm{p}=0.002)$, the body fat index $(\mathrm{p}=0.01)$ and the BMI ( $\mathrm{p}=0.01)$; however, there was no statistical difference in the heights of the soccer players and sedentary participants. Table 1 presents the results of the Student's $t$ test in terms of the physiological and biochemical variables of the two groups of participants.

The Student's $t$ test analysis showed that there was no statistical difference between the physiological measurements of the two groups in terms of the results of the ANTHR variable; however, a statistical difference was found between the ATHR, ATVL and ANTVL $(p<0.05)$. For the biochemical differences, the results of the Student's $t$ test analysis revealed no statistical difference between the two groups in terms of the levels of HDL cholesterol, vitamin B12, Hcy and hemoglobin ( $p>0.05)$; however, there was a 
statistical difference between the groups in terms of triglyceride, total cholesterol, LDL cholesterol and folate $(\mathrm{p}<0.05)$.

Table 2 presents the results of the KruskallWallis test that explored the differences between the alleles of the MTHFR C677T polymorphism.

The analysis of the physical and anthropometric differences using the KruskallWallis test showed that there was no significant difference between the alleles of the MTHFR C677T polymorphism in terms of the results of body height, mass, BMI and FFM measurements ( $>0.05)$; however, a statistical difference was found between fat percentages and age $(p<0.05)$.

The Kruskall-Wallis test was used to identify statistical differences between the alleles of the MTHFR C677T polymorphism in relation to the intensity of exercise, biochemical measurements and the HR that was measured during AT and ANT tests (Table 3).

Table 1

The results of physiological and biochemical measurements obtained from the soccer players and the sedentary group

\begin{tabular}{lccc}
\hline Group & $\begin{array}{c}\text { Sedentary } \\
(\mathrm{n}=48)\end{array}$ & $\begin{array}{c}\text { Soccer Players } \\
(\mathrm{n}=48)\end{array}$ & $p$ \\
\hline ATHR & $155.41 \pm 15.63$ & $161.68 \pm 9.07$ & $0.02^{*}$ \\
ATVL & $8.30 \pm 1.24$ & $10.31 \pm 0.94$ & $0.00^{*}$ \\
ANTHR & $174.12 \pm 12.36$ & $177.58 \pm 8.00$ & 0.11 \\
ANTVL & $10.17 \pm 1.36$ & $12.27 \pm 0.88$ & $0.00^{*}$ \\
Triglyceride $(\mathrm{mg} / \mathrm{dl})$ & $105.22 \pm 75.76$ & $7.20 \pm 33.52$ & $0.01^{*}$ \\
Total Cholesterol $(\mathrm{mg} / \mathrm{dl})$ & $166.18 \pm 29.39$ & $153.26 \pm 25.4$ & $0.03^{*}$ \\
HDL Cholesterol $(\mathrm{mg} / \mathrm{dl})$ & $37.64 \pm 7.21$ & $40.11 \pm 8.97$ & 0.15 \\
LDL Cholesterol $(\mathrm{mg} / \mathrm{dl})$ & $107.56 \pm 23.77$ & $98.31 \pm 19.35$ & $0.04^{*}$ \\
Vitamin B12 $(\mathrm{pg} / \mathrm{ml})$ & $203.89 \pm 88.95$ & $192.97 \pm 76.37$ & 0.53 \\
Folate $(\mathrm{ng} / \mathrm{ml})$ & $3.64 \pm 1.30$ & $4.53 \pm 2.48$ & $0.03^{*}$ \\
Homocysteine $(\mu \mathrm{mol} / \mathrm{L})$ & $16.99 \pm 13.28$ & $13.93 \pm 6.81$ & 0.17 \\
Hemoglobin $(\mathrm{g} / \mathrm{dL})$ & $14.63 \pm 0.73$ & $14.53 \pm 0.95$ & 0.56 \\
\hline
\end{tabular}

*Data is shown as a mean \pm standard deviation.

The intergroup analysis was carried out using the Student's t test. ${ }^{*} p<0.05$

Table 2

The results of physical and anthropometric measurements according to the alleles of the MTHFR C677T

\begin{tabular}{lccccc}
\hline & $\begin{array}{c}\mathrm{CC} \\
(\mathrm{n}=45) \\
\text { Mean Rank } \\
\text { Age }\end{array}$ & $\begin{array}{c}\mathrm{CT} \\
(\mathrm{n}=40) \\
\text { Mean Rank }\end{array}$ & $\begin{array}{c}\text { TT } \\
(\mathrm{n}=11) \\
\text { Mean Rank }\end{array}$ & $\begin{array}{c}\text { Chi- } \\
\text { Square }\end{array}$ & $p$ \\
Height $(\mathrm{cm})$ & 41.13 & 56.03 & 51.27 & 6.27 & $0.04^{*}$ \\
Body mass $(\mathrm{kg})$ & 50.50 & 48.09 & 41.82 & 0.87 & 0.64 \\
\%Fat & 45.19 & 50.58 & 54.50 & 1.36 & 0.50 \\
BMI (kg/cm) & 40.07 & 55.48 & 57.64 & 7.81 & $0.02^{*}$ \\
FFM $(\mathrm{kg})$ & 44.46 & 51.05 & 55.77 & 2.03 & 0.36 \\
\hline
\end{tabular}

The intergroup analysis was undertaken using the Kruskall-Wallis test. ${ }^{*} p<0.05$. 


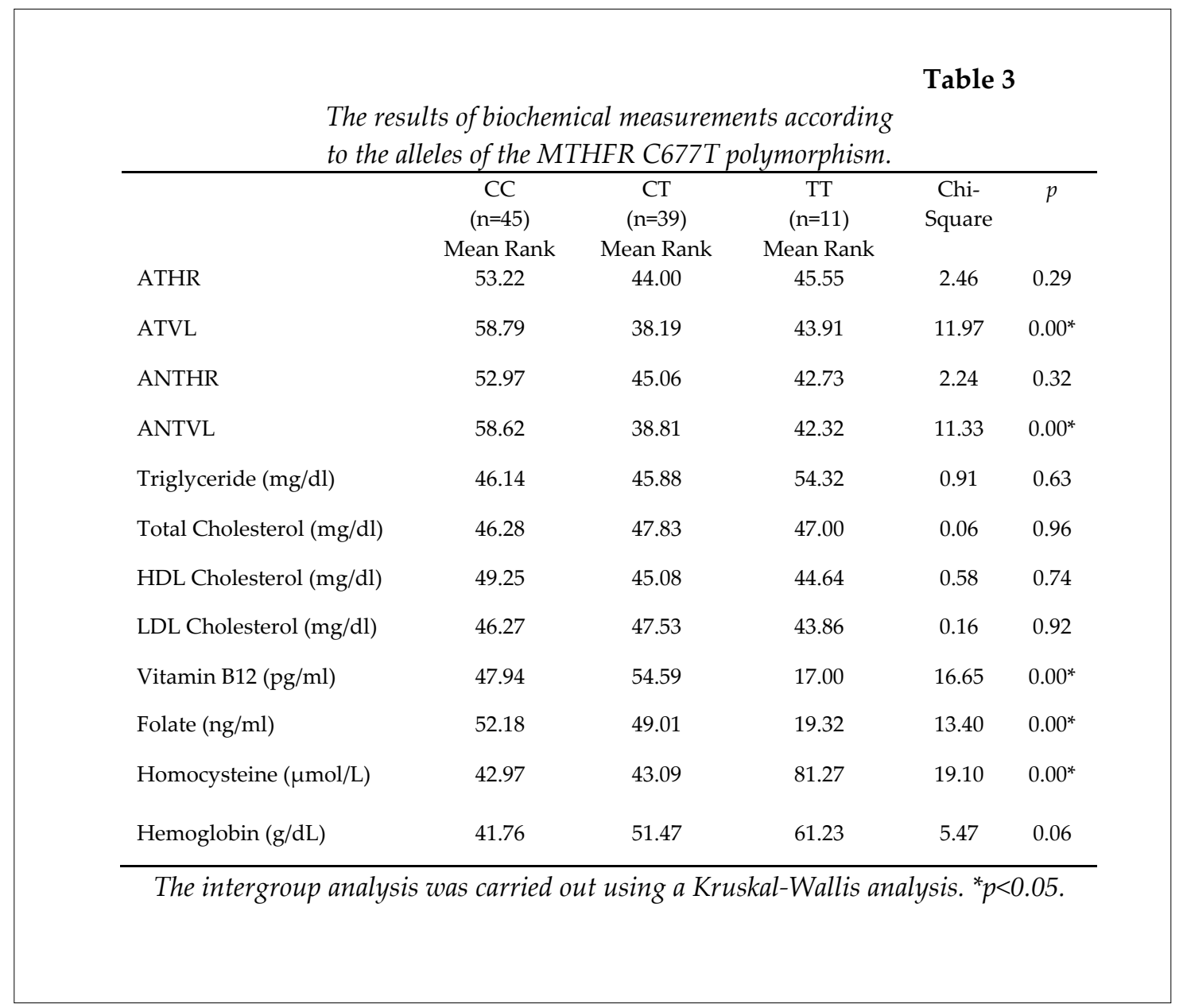

Analysis of the physiological differences using the Kruskall-Wallis test showed no significant difference between the alleles of the MTHFR C677T polymorphism in terms of the results of the ATHR and ANTHR ( $>>0.05)$; however, there was a statistically significant difference between ATVL and ANTVL $(\mathrm{p}<0.05)$.

There was no significant difference between the genotypic values of triglyceride, total cholesterol, HDL cholesterol LDL cholesterol and hemoglobin values in the MTHFR C677T polymorphism. On the other hand, the results of the current study indicated a significant decrease in vitamin B12 and folate in the TT allele group, and a significant increase in Hcy in the TT allele group.

\section{Discussion}

The physical and physiological variables of our participants indicated that, as expected, the physical and physiological variables of professional soccer players namely body mass, a body fat index, a BMI, aerobic and anaerobic thresholds were significantly higher than that of the sedentary population. In addition, when the anthropometric characteristics of our soccer players were compared with their national level French counterparts (Zouhal et al., 2013), the athletes in the current study were significantly taller, thinner and had a lower fat content. This also supports the generalization of the data taken from soccer players of the current study.

The idea that baseline cardiovascular fitness could be negatively associated with Hcy levels in healthy adolescents prompted us to study the relationship between athletic performance, homocysteine and polymorphisms of genes encoding homocysteine metabolismrelated enzymes. Therefore, we investigated the relationship between variables of physical performance and the polymorphism of the MTHFR gene which regulates plasma 
homocysteine concentration. The results of our study indicated that there was no statistically significant difference between the soccer players and healthy sedentary men in terms of HDL cholesterol, vitamin B12, hemoglobin and Hcy levels. On the other hand, Hcy values of the two groups should be carefully evaluated. According to Refsum et al. (1998), the reference value for total Hcy is $5-15 \mu \mathrm{mol} / \mathrm{L}$ in adults. In the current study, the average Hcy level of the soccer players was found to be $13.9 \mu \mathrm{mol} / \mathrm{L}$ and $16.9 \mu \mathrm{mol} / \mathrm{L}$ for the control group, which exceeded the reference level for Hcy and could be classified as a mild hyperhomocysteinemia (Heijer et al., 1998). The baseline homocysteine level and cardiovascular fitness levels of the participants may be the factors that determine the effects of exercise on the Hcy metabolism. Okura et al. (2006) showed that twenty weeks of aerobic training reduced Hcy in individuals with baseline hyperhomocysteinemia, however, Hcy slightly increased in individuals with a normal baseline level Hcy. Furthermore, in a recent study, Ruiz et al. (2007b) found that baseline cardiovascular fitness was negatively correlated with Hcy levels in female adolescents after monitoring for potential confounders including the methylenetetrahydrofolate reductase (MTHFR) $677 \quad \mathrm{C}>\mathrm{T}$ genotype. In addition, the non-existence of hyperhomocysteinemia in the athletic population can be explained by their regular training and assumed intake of ergogenic supplements, such as folate and vitamin B12 (Heijer et al., 1998; Earnest et al., 2003; Molina-Lopez, 2013). This was supported by our statistical analysis in which elevated folate levels were found to be significantly higher in soccer players than in the sedentary group.

Hyperhomocysteinemia can also occur as a result of genetic deficiencies in the enzymes of the Hcy metabolism, e.g. the MTHFR enzyme. The results of the current study showed a statistically significant difference between the MTHFR C677T polymorphism and the levels of Hcy, vitamin B12 and folate. The participants carrying the TT allele of the MTHFR C677T polymorphism had a lower level of vitamin B12 and folate than the participants carrying the CC and CT alleles. On the other hand, the Hcy levels were found to be higher in the participants carrying the TT allele of the MTHFR C677T polymorphism than those carrying the $\mathrm{CC}$ and $\mathrm{CT}$ alleles. The results of the current study are in agreement with those of other studies by Pullin et al. (2002), Husemoen (2004) and Klerk et al. (2002).

Very few homozygous individuals were found in the present study which was very similar to the distribution of the various polymorphisms of the MTHFR C677T enzyme in Gutierrez Revilla et al.'s (2004) research. The CC, CT, and TT genotype frequencies of our participants were $46.87,40.62$ and $14.45 \%$, respectively. The results of our study indicated that there were significant differences between the alleles of the MTHFR C677T polymorphism in terms of the results of the Hcy level, vitamin B12, folate, fat percentage, as well as ATVL and ANTVL variables. The Hcy level was $61 \%$ in the TT genotype, which was significantly higher than that of the CC and CT genotypes of healthy young men. The velocity attained during aerobic and anaerobic threshold tests in the homozygous CC group was efficient compared to the other two genotype groups. To our knowledge, this data is the first to show the relationship between the MTHFR C677T polymorphisms and performance variables such as the aerobic and anaerobic threshold rate in an elite athletic population. Nevertheless, the physical activity level and cardiovascular variables are the most investigated in terms of their relationship with MTHFR C677T polymorphisms (Lemitsu et al., 2010). A similar outcome was reported by Dankner et al. (2007), who found physical activity to be independently associated with lower Hcy levels. In addition, homozygotes for the TT genotype were found to have Hcy concentrations 16\% higher than those of the homozygote CC genotype individuals, controlling for B12 and folate plasma levels as well as demographic and lifestyle characteristics. Furthermore, Ruiz et al. (2007b) investigated Hcy levels in female adolescents and found that the average levels were significantly higher in the CC and CT genotypes of the MTHFR C677T polymorphism when compared with the TT genotype. On the other hand, the average Hcy levels in male adolescents of the same study were found to be significantly higher in the CT and TT genotypes when compared to the CC genotype of the MTHFR polymorphism. Also, it was found that there was a significant correlation between 
cardiovascular fitness and the levels of Hcy in female adolescents who had the MTHFR C677T polymorphism. Contrary to our findings, in another study that analyzed the MTHFR 677C $>\mathrm{T}$ genotype in children and adolescents, the authors did not observe a correlation between physical activity, fitness, body fat ratios and Hcy levels (Ruiz et al., 2007a). In a review article, Hagberg et al. (2011) used very fit subjects as an evolutionary "control group" and proposed that higher cardiovascular fitness levels protected against the effects of the high-risk MTHFR TT genotype on carotid stiffness. They also implied that the cardiovascular risk genotype expressed itself only when the environmental trigger of low cardiovascular fitness was present.

With regard to other variables of the current study, no statistically significant differences were found between the MTHFR C677T polymorphism and the levels of hemoglobin, total cholesterol, HDL cholesterol and LDL cholesterol. On the contrary, Fortunato et al. (2007) carried out a study including healthy female participants who engaged in regular exercise and those carrying the homozygote TT genotype of the MTHFR C677T polymorphism were found to have a lower level of hemoglobin than subjects with the CC and CT genotypes. Also, Gil-Prieto et al. (2009) found that the prevalence of hyperhomocysteinemia was seen to increase with the MTHFR C677T mutant allele. In addition to the TT polymorphism, a serum folate and HDL deficit reduces the ability to metabolize homocysteine. The existence of the genotype TT of the MTHFR gene and higher Hcy values showed significantly higher values of plasma HDL-C. Westerbuch et al. (2001) explained the Hcy increase with the homocysteine-induced endoplasmic reticulum stress that was connected to important regulatory proteins of cholesterol and triglyceride synthesis and uptake. The reason for the controversy between literature and our study in terms of blood lipids and the MTHFR polymorphism could be the Hcy characteristics of the study populations. In the literature, most of the studies (Gil-Prieto et al., 2009; Westerbuch et al., 2001) were carried out in a hyperhomocystenic population. On the other hand, the Hcy range of our study was broad, as soccer players were found to be in the normal range of Hcy values; however, the sedentary group could be classified as having mild hyperhomocysteinemia. Zhang et al. (2010) also showed that there were effects on their populations in terms of the relationship between the allelic frequencies of MTHFR C677T and serum TC, TG and LDL-C levels.

\section{Conclusion}

Significant differences were found between the alleles of the MTHFR C677T polymorphism in terms of the Hcy level, vitamin B12, folate, the fat percentage, aerobic and anaerobic threshold rates. To our knowledge, this data is the first to show the relationship between the MTHFR C677T polymorphisms and performance variables such as the aerobic and anaerobic threshold rate.

\section{References}

Andreassi MG, Botto N, Cocci F, Battaglia D, Antonioli E, Masetti S, Manfredi S, Colombo MG, Biagini A, Clerico A. Methylenetetrahydrofolate reductase gene C677T polymorphism, homocysteine, vitamin B12, and DNA damage in coronary artery disease. Hum Genet, 2003; 112: 171-177

Borch KW, Ingjer F, Larsen S, Tomten SE. Rate of accumulation of blood lactate during graded exercise as a predictor of 'anaerobic threshold'. J Sports Sci, 1993; 11: 49-55

Dankner R, Chetrit A, Dror GK, Sela BA. Physical activity is inversely associated with total homocysteine levels, independent of C677T MTHFR genotype and plasma B vitamins. AGE, 2007; 29: 219-227

Earnest C, Wood KA, Church TS. Complex multivitamin supplementation improves homocysteine and resistance to LDL-C oxidation. J Am Coll Nutr, 2003; 22(5): 400-407

Fletcher O, Kessling AM. MTHFR association with arteriosclerotic vascular disease? Hum Genet, 1998; 103: $11-21$ 
Fortunato G, Fattoruso O, Cateria MD, Mancini A, Di Flore R, Alfieri A, Tafuri D, Buono P. RAS and MTHFR gene polymorphisms in a healthy exercise-trained population: association with the MTHFR (TT) genotype and a lower hemoglobin level. Int J Sports Med, 2007; 28(2): 172-7

Gil-Prieto R, Hernández V, Cano B, Oya M, Gil A. Plasma homocysteine in adolescents depends on the interaction between methylenetetrahydrofolate reductase genotype, lipids and folate: a seroepidemiological study. Nutr Metab (Lond), 2009; Oct 5; 6: 39. doi: 10.1186/1743-7075-6-39

Gutierrez Revilla JI, Perez HF, Tamparillas SM, Calvo Martin MT. Influence of biochemical and genetic factors on homocysteine concentrations. An Pediatr (Barc), 2004; 60(3): 215-21

Hagberg JM, Rankinen T, Loos R, Pérusse L, Roth SM, Wolfarth B, Bouchard C. Advances in exercise, fitness, and performance genomics in 2010. Med Sci Sports Exerc, 2011 May; 43(5): 743-752

Heijer MD, Brouwer IA, Bos Gerard MJ, Blom HJ, Van der Put NMJ, Spaans AP, Rosendaal FR, Thomas CMG, Haak HL, Wijermans PW, Gerrits WJ. Vitamin supplementation reduces blood homocysteine levels: a controlled trial in patients with venous thrombosis and healthy volunteers. Arterioscler Thromb Vasc Biol, 1998; 18: 356-361

Husmoen LLN, Thomsen TF, Fenger M, Jorgensen T. Effect of lifestyle factors on plasma total homocysteine concentrations in relation to MTHFR(C677T) genotype. Inter99 (7). Eur J Clin Nutr, 2004; 58: 1142-1150

Husemoen LLN, Thomsen TF, Fenger M, Jorgensen T. Changes in lifestly and total homocysteine in relation to MTHFR (C677T) genotype. Inter99 (7). Eur J Clin Nutr, 2006; 60: 614-622

Klerk M, Verhoef P, Clarke R, Blom HJ, Kok FJ, Schounten EG. MTHFR 677C $\rightarrow$ T polymorphism and risk of coronary heart disease: a meta-analysis. JAMA, 2002; 288: 2023-2031

Lemitsu M, Murakami H, Sanada K, Yamamoto K, Kawano H, Gando Y, Miyachi M. Lack of carotid stiffening associated with MTHFR 677TT genotype in cardiorespiratory fit adults. Physiol Genomics, 2010; 42(2): 259-65

Mennen LI, Courcy GP, Guilland JC, Ducros V, Bertrais S, Nicolas JP, Maurel M, Zarebska M, Favier A, Franchisseur C, Hercberg S, Galan P. Homocysteine, cardiovascular disease risk factors, and habitual diet in the French Supplementation with Antioxidant Vitamins and Minerals Study. Am J Clin Nutr, 2002; 76: 1279-1289

Molina-López J, Molina JM, Chirosa LJ, Florea DI, Sáez L, Planells E. Effect of folic acid supplementation on homocysteine concentration and association with training in handball players. J Int Soc Sports Nutr, 2013 Feb 21; 10(1):10. doi: 10.1186/1550-2783-10-10

Okura T, Rankinen T, Gagnon J, Lussier-Cacan S, Davignon J, Leon AS, Rao DC, Skinner JS, Wilmore JH, Bouchard C. Effect of regular exercise on homocysteine concentrations: the HERITAGE Family Study. Eur J Appl Physiol, 2006; 98: 394-401

Pullin CH, Wilson JF, Ashfield-Watt PAL, Clark ZE, Whiting JM, Lewis MJ, McDowell LFW. Influence of methylenetetrahydrofolate reductase genotype, exercise and other risk factors on endothelial function in healthy individuals. Clinical Sci, 2002; 102: 45-50

Rasmussen LB, Ovesen L, Bülow İ, Knudsen N, Laurberg P, Perrild H. Folate intake, lifestyle factors and homocysteine concentrations in younger and older women. Am J Nutr, 2000; 72: 1156-1163

Refsum H, Ueland PM, Nygard O, Vollset SE. Homocysteine and cardiovascular disease. Annu Rev Med, 1998; 49: 31-62

Ruiz JR, Hurting-Wennlöf A, Ortega FB, Patterson E, Nilson TK, Costillo MJ, Sjöström M. Homocysteine levels in children and adolescents are associated with the methylenetetrahydrofolate reductase $677 \mathrm{C}>$ T genotype, but not with physical activity, fitness or fatness: The European Youth Heart Study. Brit J Nutr, 2007a; 97: 255-262

Ruiz JR, Sola R, Gnzales-Gross M, Ortega FB, Vicente-Rodriguez G, Garcia-Fuentes M, Gutierrez A, Sjöström M, Pietrzik K, Casillo MJ. Cardiovascular fitness is negatively associated with homocysteine levels in 
female adolescents. Arch Pediatr Adolesc Med, 2007b; 161: 166-171

Ueland M, Hustad S, Schneede J, Refsum H, Vollset SE. Biological and clinical implications of the MTHFR C677T polymorphism. Trends Pharmacol Sci, 2001; 22(4): 195-201

Weisberg I, Tran P, Christensen B, Sibani S, Rozen R. A second genetic polymorphism in methylenetetrahydrofolate reductase (MTHFR) associated with decreased enzyme activity. Mol Genet Metab, 1998; 64: 169-172

Werstuck GH, Lentz SR, Dayal S, Hossain GS, Sood SK, Shi YY, Zhou J, Maeda N, Krisans SK, Malinow MR, Austin RC. Homocysteine-induced endoplasmic reticulum stress causes dysregulation of the cholesterol and triglyceride biosynthetic pathways. J Clin Invest, 2001 May; 107(10): 1263-73

Zhang L, Xing Yin R, Ying Liu W, Miao L, Feng Wu D, Htet Htet Aung L, Jiang Hu X, Li Cao X, Zhen Wu J. Shang-Ling Pan. Association of methylenetetrahydrofolate reductase C677T polymorphism and serum lipid levels in the Guangxi Bai Ku Yao and Han populations. Lipids in Health Dis, 2010; 9: 123

Zouhal H, Lemoal E, Wong del P, Benounis O, Castagna C, Duluc C, Owen AL, Drust B. Physiological responses of general vs. specific aerobic endurance exercises in soccer. Asian J Sports Med, 2013 Sep; 4(3): 213-20

\section{Corresponding author:}

\section{Selda Bereket Yücel}

Marmara Üniversitesi Beden Eğitimi ve Spor Yüksek Okulu

Anadolu Hisarı Yerleşkesi, Beykoz-İstanbul/Turkey

Phone: (532) 664-1686

E-mail: seldabereket@hotmail.com 